\title{
Concentration and purification by magnetic separation of the erythrocytic stages of all human Plasmodium species Clotilde Ribaut $^{1}$, Antoine Berry ${ }^{2,3}$, Séverine Chevalley ${ }^{1}$, Karine Reybier ${ }^{1}$, Isabelle Morlais ${ }^{3}$, Daniel Parzy ${ }^{4}$, Françoise Nepveu ${ }^{1}$, Françoise Benoit-Vical2,5 and Alexis Valentin*1
}

\begin{abstract}
Address: ${ }^{1}$ Université de Toulouse, Laboratoire Pharmacochimie des Substances Naturelles et Pharmacophores Redox, UMR 152 IRD-Université Université Paul Sabatier, Faculté de Pharmacie, 118 route de Narbonne, 31062 Toulouse Cedex 9, France, ${ }^{2}$ Université de Toulouse, Service de Parasitologie et Mycologie, Hôpital Rangueil-Larrey CHU de Toulouse et Faculté de Médecine de Rangueil, 1 av. Jean Poulhes - TSA 50032, 31059 Toulouse cedex 9, France, ${ }^{3}$ Laboratoire d'entomologie médicale, OCEAC-IRD, BP288, Yaoundé, Cameroon, ${ }^{4}$ Unité de Parasitologie, IMTSSA, 13998 Marseille Armées, France and ${ }^{5}$ Laboratoire de Chimie de Coordination du CNRS, UPR8241, 31077 Toulouse 4, France

Email: Clotilde Ribaut - ribaut@cict.fr; Antoine Berry - antoine.berry@yahoo.fr; Séverine Chevalley - severine.maurel@cict.fr; Karine Reybier - reybier@cict.fr; Isabelle Morlais - morlais@ird.fr; Daniel Parzy - d.parzy@free.fr; Françoise Nepveu - nepveu@cict.fr; Françoise Benoit-Vical - Francoise.Vical@toulouse.inserm.fr; Alexis Valentin* - valentin@cict.fr

* Corresponding author
\end{abstract}

Published: 5 March 2008

Malaria Journal 2008, 7:45 doi:10.1 186/1475-2875-7-45

This article is available from: http://www.malariajournal.com/content/7/I/45

(c) 2008 Ribaut et al; licensee BioMed Central Ltd.

This is an Open Access article distributed under the terms of the Creative Commons Attribution License (http://creativecommons.org/licenses/by/2.0), which permits unrestricted use, distribution, and reproduction in any medium, provided the original work is properly cited.
Received: 21 December 2007

Accepted: 5 March 2008

\begin{abstract}
Background: Parasite concentration methods facilitate molecular, biochemical and immunological research on the erythrocytic stages of Plasmodium. In this paper, an adaptation of magnetic MACS ${ }^{\circledR}$ columns for the purification of human Plasmodium species is presented. This method was useful for the concentration/purification of either schizonts or gametocytes.

Results and conclusions: The magnetic removal of non-parasitized red blood cells (in vivo and in vitro) using magnetic columns (MACS) was evaluated. This easy-to-use technique enriched schizonts and gametocytes from Plasmodium falciparum in vitro cultures with a very high degree of purity. In addition, all haemozoin-containing stages (schizonts and/or gametocytes) from the peripheral blood of infected patients could be concentrated using this method. This method is particularly useful for the concentration of non-falciparum species, which do not grow in culture and are otherwise difficult to obtain in large amounts.
\end{abstract}

\section{Background}

Falciparum malaria is one of the most common diseases in tropical countries. There are about 300 million new cases, and more than two million deaths due to this illness every year [1]. Different methods have been described to isolate and/or purify living parasites from in vitro cultures or from murine Plasmodium $[2,3]$. However, non-falciparum species of human Plasmodium (namely Plasmodium vivax, Plasmodium ovale, and Plasmodium malariae) are not easy to purify or concentrate from the blood of infected patients. Difficulties in producing large amounts of parasite material from the blood stages of Plasmodium sp. have restricted progress in research on non-falciparum malaria, but also studies on gametocyte biology. Moreover, in endemic regions, the use of an easy single-step method for the purification and/or concentration of the gametocytes could be useful for biological and epidemiological studies [4]. The property of all human 
Plasmodium species to degrade haemoglobin (an Fe(II) diamagnetic complex) into haemozoin (an $\mathrm{Fe}(\mathrm{III})$ paramagnetic complex) was used, making possible the magnetic purification of parasitized red blood cells containing haemozoin. The present study demonstrates the high degree of purity that can be obtained for the synchronization of in vitro cultures of Plasmodium falciparum, either on asexual or sexual erythrocytic stages, and the usefulness of magnetic separation for the enrichment and purification of Plasmodium parasitized red blood cells from infected malaria patients.

\section{Methods}

In vitro culture of Plasmodium falciparum

Parasites were cultured according to the method described by Trager and Jensen [5], with modifications [6]. Briefly, parasites were maintained in human erythrocytes $(\mathrm{O} \pm$, Blood Bank, EFS, Toulouse, France) routinely at $0.5-4 \%$ parasitaemia in culture medium (haematocrit: $2-4 \%$ ). The culture medium consisted of RPMI (Cambrex, Belgium) complemented with $25 \mathrm{mM}$ Hepes (Cambrex) and $2 \mathrm{mM}$ glutamine (Sigma, l'Isle d'Abeau, France) and supplemented with $7.5 \%$ human serum (EFS). The knob+ strains (FcB1-Colombia, W2-Indochina and F32-Tanzania) were concentrated by flotation with Plasmion ${ }^{\circledast}$ (Fresenius Kabi France) followed by 5\% D-sorbitol (Sigma) lysis [2]. The knobby-strain (FcM29-Cameroon) was only synchronized by $5 \%$ D-sorbitol lysis every $48 \mathrm{hrs} \mathrm{[7].}$

Gametocyte cultures of strain W2 were initiated as described elsewhere [8], with minor modifications [9]. Cultures were then treated with $50 \mathrm{mM} \mathrm{N}$-acetyl-D-glucosamine (Sigma) for 3-5 days to remove most of the asexual stages. Young (stage II, 7-day-old) or old (stage IV-V, 13-day-old) gametocyte cultures were tested for magnetic enrichment.

\section{Parasitized blood from patients}

Parasitized blood from patients presenting symptoms of malaria was obtained by venipuncture for diagnosis purposes in the University Hospital in Toulouse. One EDTAtube was routinely filled. Diagnosis (malaria, Plasmodium species and parasitaemia) was made by $\mathrm{QBC}^{\circledast}$ associated to thin smear and the Plasmodium species and single infection was confirmed by routine real-time PCR [10]. When a non-falciparum malaria was diagnosed or when gametocytes (whatever the Plasmodium species) were found, the remaining blood was used for the concentration of the parasites with magnetic columns. Before passage on columns, the blood cells were washed (centrifugation at 800 $\mathrm{g}, 10 \mathrm{~min}$ ) once in 10 volumes of warmed culture PBS (Cambrex). The enrichment of $P$. falciparum gametocytes was also carried out on blood from Cameroonian patients with diagnosed malaria (IRD-OCEAC, Yaounde, Cameroon). In every case, the blood was washed once with
PBS without human serum before passage on the columns.

Each sample from Toulouse University Hospital patients corresponded to an EDTA-tube bottom that was used for malaria diagnosis. Experiments did not need any additional blood sample. Blood samples were anonymized and any remaining material was discarded in order to respect the confidentiality of participants and protection of personal data. Protocol for gametocyte carrier recruitment received ethical approval from the National Ethic Committee in Cameroon (039/CNE/MP/06).

For both sampling sites (France and Cameroon), trophozoite parasitaemia was evaluated on thin smears while a gametocyte count was performed on calibrated $5 \mu \mathrm{L}$ thick films before the enrichment was carried out. For some falciparum-infected patients from Cameroon, blood samples, with no evidence for the presence of gametocytes on thick films were also treated with magnetic columns for gametocyte enrichment.

\section{Purification}

Whether from culture or from patients, parasitaemia in infected erythrocytes was evaluated by two experienced technicians before any experimentation. Prior to purification, the $\mathrm{MACS}^{\circledast}$ (25 LD columns, Miltenyi Biotec, Germany) columns were filled with warmed $\left(37^{\circ} \mathrm{C}\right) \mathrm{RPMI}$ or PBS. When the purification was carried out in order to synchronize the culture, the experimentation was performed under sterile conditions. The test blood (from culture or from patients) was then deposited on the top of the column (typically, $1 \mathrm{~mL}$ at $25-50 \%$ haematocrit) which was held in a Quadro MACS ${ }^{\circledast}$ magnetic support. Warmed $\left(37^{\circ} \mathrm{C}\right)$ culture medium was then added until the eluent was apparently free of red blood cells. The column was removed from the magnetic support and a further $4 \mathrm{~mL}$ (dead volume of the column) of culture medium added and the eluent recovered. This eluent was then centrifuged $(800 \mathrm{~g}, 10 \mathrm{~min})$ and the supernatant was discarded. The pellet was used to prepare blood smears and calibrated $(5 \mu \mathrm{L})$ thick films, both stained with Giemsa.

All images were digitally registered with a Nikon digital camera (DXM1200) mounted on a Nikon microscope (Eclipse E 400) and not modified with any software. Visual counting of gametocytes was performed on Giemsastained smears before and after concentration.

\section{Results and discussions Concentration and synchronization of $P$. falciparum schizonts}

As $P$. falciparum schizonts contained paramagnetic Fe(III) species, they were highly purified by the MACS ${ }^{\circledast}$ columns. 
Table I: Enrichment of $P$. falciparum from in vitro cultures (comparison between Plasmion ${ }^{\circledR}$, a gelatine solution, and magnetic columns)

\begin{tabular}{cccc}
\hline \multicolumn{3}{c}{ Parasitaemia (\%) $^{\mathrm{a}}$} \\
\hline Strain & Initialc & Plasmion & \\
& & & Magnetic columns \\
\hline FcBI (K+) & 2.2 & $14.8(6.7)^{\mathrm{c}}$ & $98(44)$ \\
FcM29 (K-) & 2.8 (Fig. A) & $3.4(1.2)$. & $96(34)($ Fig. B) \\
F32 (K+) & 3.1 & $13(4.2)$ & $91(29)$ \\
W2 (K+) & 2.1 & $11(5.2)$ & $94(44)$ \\
\hline
\end{tabular}

a: parasitaemia was evaluated by visual estimation and counting of at least 2,000 erythrocytes

b: presence $(\mathrm{K}+)$ or absence $(\mathrm{K}-)$ of knobs on erythrocyte membrane c: in brackets, relative enrichment of the technique (parasitaemia after enrichment/initial parasitaemia)

Table 1 summarizes the results obtained for $P$. falciparum cultured in vitro. Clearly, MACS ${ }^{\circledast}$ enabled $P$. falciparum infected erythrocytes to be purified when containing paramagnetic iron(III) species (Figures 1A and 1B). This synchronization was efficient either with knobby $(\mathrm{K}+)$ or with knobless (K-) strains while and, as expected, only $\mathrm{K}+$ strains were enriched with Plasmion ${ }^{\oplus}$. The yield of purity after magnetic enrichment was higher than $90 \%$, whatever the strain tested. Such a schizont-enriched culture can be very usefull for biochemical or molecular analysis [11]. This technique, when performed under sterile conditions and in a $37^{\circ} \mathrm{C}$ incubator, allowed a highly efficient synchronization of $P$. falciparum from in vitro cultures and rapid recovery of a highly synchronized parasite population. Moreover, it can be used to determine the point of action of a potential antimalarial drug [9].

\section{Concentration of $\mathrm{P}$. falciparum gametocytes}

As $P$. falciparum gametocytes also contained paramagnetic iron, it was of interest to test the ability of the MACS ${ }^{\circledast}$ columns to purify them, either from in vitro cultures or from the peripheral blood of Plasmodium infected patients. Moreover, as gametocytes are not available in large amounts in blood or in culture, a highly purified population should be of interest for biochemical and/or molecular purposes. The use of $\mathrm{MACS}^{\circledast}$ columns to improve the synchronous production of $P$. falciparum gametocytes in vitro has already been reported, but without any indication of the yield [12]. When tested on in vitro gametocyte culture, enrichment at a very high yield (enrichment rate higher than $100 \times$ ) was obtained, whatever the gametocyte stage (II to IV) tested (see Table 2 and Figure 1E). Given this result, it was of interest to evaluate the efficiency of $\mathrm{MACS}^{\circledast}$ columns for gametocyte enrichment in the field. With infected peripheral blood we obtained a relative enrichment of gametocytes ranging from 43 to 85 (Table 2 and Figures 1C and 1D). The results presented here only concerns three different patients, but a very high enrichment (at least more than 40 fold) was obtained with all the other patients tested. For P. falciparum infected blood,
MACS $^{\circledast}$ columns enabled us to separate trophozoites (which did not contained haemozoin and were found in the first eluent) and gametocytes from the same donor with a very high reliability. Less than one parasite stage mismatch/1,000. This result allowed unambiguous biological or molecular studies on separate stages of the parasite from the same patient. In $P$. falciparum infection, the peripheral blood contains only trophozoites and the gametocyte stages IV and V, while schizonts and early gametocyte stages (I and II) are sequestered [13].

\section{Non-falciparum purification}

Peripheral blood containing non-falciparum Plasmodium was also tested for enrichment with MACS ${ }^{\circledast}$. Whatever the species tested, the peripheral blood was enriched in erythrocytic stages (Table 3 and Figures $1 \mathrm{~F}$ to $1 \mathrm{H}$ ). Because in non-falciparum malaria all stages were in the blood circulation, the enrichment concerned all haemozoin-containing parasites (namely schizonts, Figure $1 \mathrm{H}$ and gametocytes Figure 1F) and led to high levels of enrichment (Table 3).

This is the first time that an easy-to-use and fast method has been described for human non-falciparum Plasmodium enrichment. The MACS ${ }^{\varpi}$ columns, in the field, could be an efficient way to concentrate parasites and could associate, in one step, the advantages of thin smears and thick films with species diagnosis and enrichment. In research laboratories, the purification of non-falciparum Plasmodium in large enough amounts to work on specific pathways or to improve in vitro cultures [14] could also be facilitated by this material.

In conclusion, the simple one-step MACS $^{\circledast}$ columns extended here to all the human Plasmodium species allowed large-scale concentration and purification of different parasite stages, with emphasis on gametocytes from $P$. falciparum from both in vitro cultures and from Plasmodium-infected peripheral blood. This method offers opportunities to extend knowledge on the biology of Plasmodium sp.

\section{Authors' contributions}

CR: Initiated falciparum purification, realized the experimental conditions and contributed to the preparation of the manuscript. AB: developed of the protocols and carried out most parts of the laboratory experiments for gametocyte carrier experiments, and contributed to the preparation of the manuscript. SC: maintained in vitro culture, produced gametocytes, concentrated parasites and helped in analysing results. $\mathrm{KR}$, : was implicated in elaboration of the experimental conditions, contribution to the preparation of the manuscript. IM: was responsible in Cameroon for supervision of IRD/OCEAC laboratory work and protocol for gametocyte carrier recruitment. DP: 

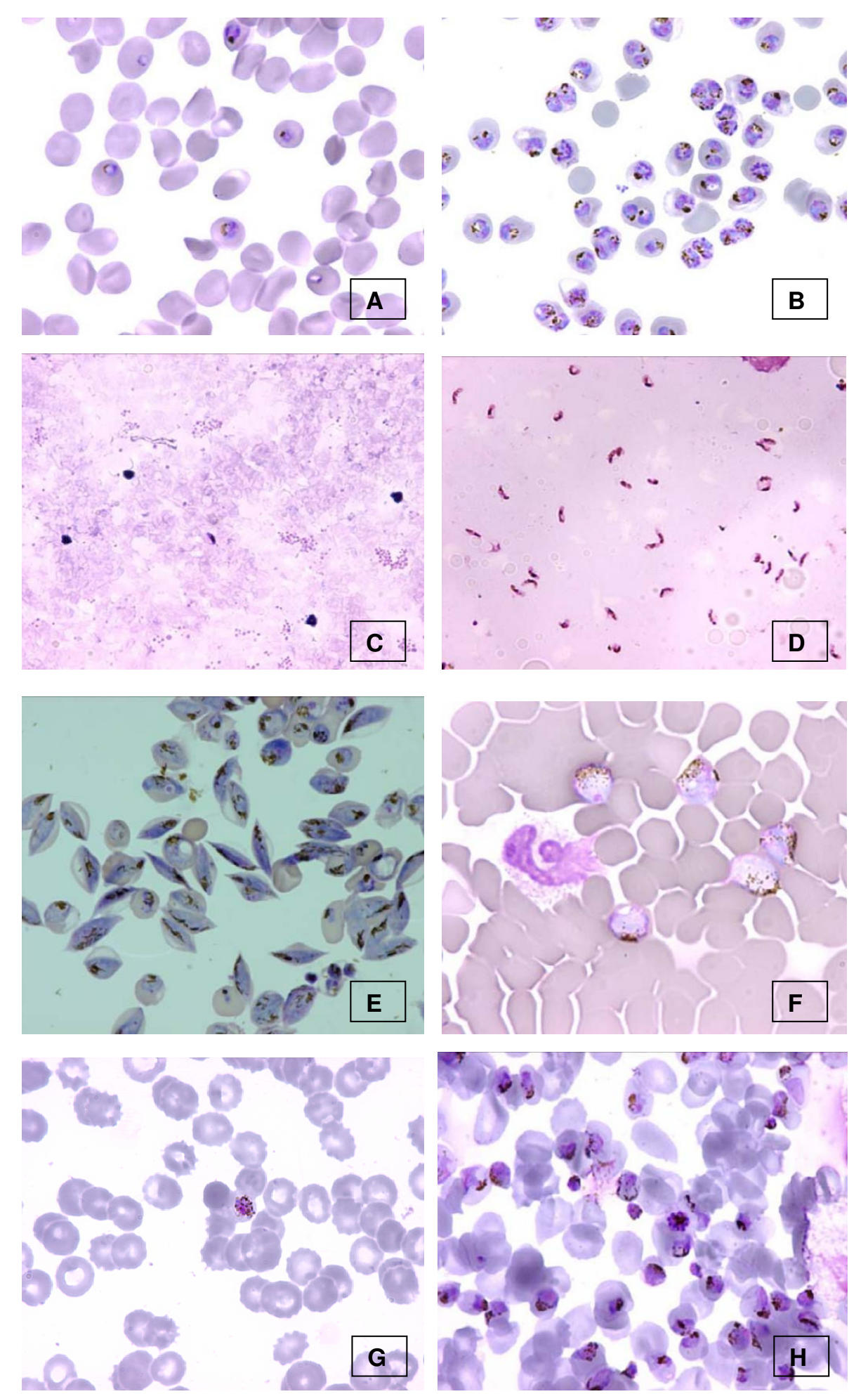

Figure I

Plasmodium purification with MACS ${ }^{\circledR}$ (magnification $\times 100$, except $C$ and D: $\left.\times 40\right)$ P. falciparum in vitro culture before $(A)$ and after (B) purification (thin smears). Plasmodium falciparum gametocyte containing blood before (C) and after (D) purification (calibrated thick film). Plasmodium falciparum gametocytes obtained from in vitro culture (E). Plasmodium ovale gametocytes from an infected patient $(F)$. Plasmodium malariae schizonts before $(G)$ and after $(H)$ purification (thin smears) 
Table 2: Enrichment of gametocytes obtained with $P$. falciparum from infected blood from patients and in vitro cultures

\begin{tabular}{ccccc}
\hline & Initial $^{\mathrm{a}}$ & Eluent $^{\mathrm{b}}$ & Final count $^{\mathrm{c}}$ & Enrichment rate $^{\mathrm{d}}$ \\
\hline Patients bloode & 21 & 2 & 1120 & 53 \\
& 6 & 1 & 514 & 85 \\
& 19 (Fig. C) & 1 & 819 (Fig. D) & 43 \\
In vitro culture & $0.8 \pm 0.03 \% \mathrm{f}$ & $<0.01$ & $91 \%$ & 114 \\
Stage II-III & $0.9 \pm 0.1 \%$ & $<0.01$ & $88 \%$ & 98 \\
In vitro culture Stage IV-V & $0.4 \pm 0.02 \%$ & $<0.01$ & $95 \%$ & 237 \\
& $0.8 \pm 0.1 \%$ & $<0.01$ & $92 \%$ & 115 \\
& $0.7 \% \pm 0.1 \%$ & $<0.01$ & $94 \%$ (Fig. I-E) & 134 \\
\hline
\end{tabular}

a: calibrated thick films $(5 \mu \mathrm{L})$, gametocyte count

b: calibrated thick films $(5 \mu \mathrm{L})$, gametocyte count after elution from the columns

c: Calibrated thick films $(5 \mu \mathrm{L})$, gametocyte count on the retained

blood

$\mathrm{d}$ : calculated as ratio of final/initial counts

e: estimated on three patients with presence of gametocytes

f: estimated on thin blood smears (at least 10,000 red blood cells were counted).

assisted in redaction. FN: Head of the laboratory, discussion and assisted in redaction. FBV, initiated the falciparum gametocyte production, discussion concerning redaction and contributed to the preparation of the manuscript. AV: Redaction, non-falciparum purification, falciparum gametocytes purification, pictures. All authors read and approved the final manuscript.

\section{Acknowledgements}

The authors thank the European Commission for support (RTD-Project 6th FP, Contract N018602, Read-UP). We thank Eliane Pélissou, Martine Boyer, Annie Monnier, Brigitte Vielcastel and Sylvie Kemleu-Zebazse for technical assistance. We also thank Dr Engelbert Manga, head of the district hospital of Mfou (Cameroun), for the supervision of the gametocyte carrier recruitment and John Woodley for editing the English.

Table 3: Enrichment of non-falciparum parasitized erythrocytes

\begin{tabular}{|c|c|c|c|}
\hline \multirow[b]{2}{*}{ Species } & & \multicolumn{2}{|c|}{ Parasitaemia (\%) } \\
\hline & & Initial: & $\begin{array}{l}\text { After magnetic } \\
\text { columns }\end{array}$ \\
\hline \multirow[t]{2}{*}{ P. malariae ${ }^{\mathrm{a}}$} & $\begin{array}{l}\text { Trophozoites and } \\
\text { schizonts }\end{array}$ & $<0.1$ & 12 \\
\hline & & 0.3 (Fig. G) & 17 (Fig. H)b \\
\hline \multirow[t]{2}{*}{ P. ovale } & $\begin{array}{l}\text { Trophozoites and } \\
\text { schizonts }\end{array}$ & $<0.1$ & 15 \\
\hline & $\begin{array}{l}\text { Trophozoites } \\
\text { schizonts and } \\
\text { gametocytes }\end{array}$ & $\begin{array}{l}<0.01 \text { and } \\
\text { presence of } \\
\text { gametocytes }\end{array}$ & $\begin{array}{l}6, \text { and } \\
\text { numerous } \\
\text { gametocytes } \\
\text { (Fig. F) }\end{array}$ \\
\hline P. vivax & $\begin{array}{l}\text { Trophozoites and } \\
\text { schizonts }\end{array}$ & $<0.1$ & 16 \\
\hline
\end{tabular}

a: species and mono-infestation were confirmed by PCR

b: enrichment was performed 36 hours after blood collection

\section{References}

I. Snow RW, Guerra CA, Noor AM, Myint HY, Hay SI: The global distribution of clinical episodes of Plasmodium falciparum malaria. Nature 2005, 434(7030):214-217.

2. Lelievre J, Berry A, Benoit-Vical F: An alternative method for Plasmodium culture synchronization. Exp Parasitol 2005, 109(3): 195-197.

3. Trang DT, Huy NT, Kariu T, Tajima K, Kamei K: One-step concentration of malarial parasite-infected red blood cells and removal of contaminating white blood cells. Malar J 2004, 3:7.

4. Nassir E, Abdel-Muhsin AM, Suliaman S, Kenyon F, Kheir A, Geha H, Ferguson HM, Walliker D, Babiker HA: Impact of genetic complexity on longevity and gametocytogenesis of Plasmodium falciparum during the dry and transmission-free season of eastern Sudan. Int J Parasitol 2005, 35(I):49-55.

5. Trager W, Jensen JB: Human malaria parasites in continuous culture. Science 1976, 193(4254):673-675.

6. Jacquemond-Collet I, Benoit-Vical F, Valentin A, Stanislas E, Mallie M, Fouraste I: Antiplasmodial and cytotoxic activity of galipinine and other tetrahydroquinolines from Galipea officinalis. Planta Med 2002, 68(I):68-69.

7. Lambros C, Vanderberg JP: Synchronization of Plasmodium falciparum erythrocytic stages in culture. J Parasitol 1979, 65(3):418-420.

8. Ifediba T, Vanderberg JP: Complete in vitro maturation of Plasmodium falciparum gametocytes. Nature 1981, 294(5839):364-366.

9. Benoit-Vical F, Lelievre J, Berry A, Deymier C, Dechy-Cabaret O, Cazelles J, Loup C, Robert A, Magnaval JF, Meunier B: Trioxaquines are new antimalarial agents active on all erythrocytic forms, including gametocytes. Antimicrob Agents Chemother 2007, 5I(4): 1463-1472.

10. Berry A, Fabre R, Benoit-Vical F, Cassaing S, Magnaval JF: Contribution of PCR-based methods to diagnosis and management of imported malaria. Med Trop (Mars) 2005, 65(2):176- 83.

II. Uhlemann AC, Staalsoe T, Klinkert M, Hviid L: Analysis of Plasmodium falciparum-infected red blood cells. MACS \& More 2000 , 4:7-8.

12. Fivelman QL, McRobert L, Sharp S, Taylor CJ, Saeed M, Swales CA, Sutherland CJ, Baker DA: Improved synchronous production of Plasmodium falciparum gametocytes in vitro. Mol Biochem Parasitol 2007, 154(1): I19-123.

13. Alano P: Plasmodium falciparum gametocytes: still many secrets of a hidden life. Mol Microbiol 2007.

14. Udomsangpetch R, Somsri S, Panichakul T, Chotivanich K, Sirichaisinthop J, Yang Z, Cui L, Sattabongkot J: Short-term in vitro culture of field isolates of Plasmodium vivax using umbilical cord blood. Parasitol Int 2007, 56(I):65-69.

\begin{tabular}{|} 
Publish with BioMed Central and every \\
scientist can read your work free of charge \\
"BioMed Central will be the most significant development for \\
disseminating the results of biomedical research in our lifetime." \\
Sir Paul Nurse, Cancer Research UK \\
Your research papers will be: \\
• available free of charge to the entire biomedical community \\
• peer reviewed and published immediately upon acceptance \\
• cited in PubMed and archived on PubMed Central \\
• yours - you keep the copyright \\
Submit your manuscript here: \\
http://www.biomedcentral.com/info/publishing_adv.asp
\end{tabular}

